Recepción: 24 / 07 / 2018

Aceptación: 27 / 08 / 2018

Publicación: 15 / 09 / 2018

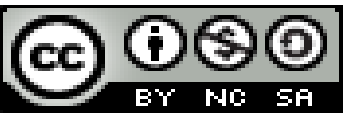

Ciencias económicas y empresariales Artículo de investigación

\title{
Determinantes del modelo estratégico publicitario en el comportamiento del consumidor de las empresas sector comercial
}

\author{
Determinants of the advertising strategic model in business consumer behavior \\ commercial sector
}

\section{Determinantes do modelo estratégico de publicidade no setor comercial de comportamento do consumidor empresarial}

\author{
Carmen I. Zambrano-Salazar ${ }^{\text {I }}$ \\ ivanova_love2@hotmail.com \\ Leonardo G. Ballesteros-López II \\ leonardogballestros@uta.edu.ec \\ María C. Abril-Freire ${ }^{\mathrm{III}}$ \\ mariacabril@uta.edu.ec \\ César A. Guerrero-Velástegui ${ }^{\text {IV }}$ \\ ca.guerrero@uta.edu.ec
}

\section{Correspondencia: ivanova_love2@hotmail.com}

\footnotetext{
${ }^{\text {I }}$ Docente de la Universidad Técnica de Ambato, Ambato, Ecuador.

${ }^{\text {II }}$ Magíster en Gestión Estratégica Empresarial MBA, Ingeniero Comercial, Licenciado en Ciencias Administrativas Administrador en Mercadotecnia, Docente de la Universidad Técnica de Ambato, Ambato, Ecuador.

III Magíster en Administración de Empresas Mención Planeación, Magíster en Gestión del Talento Humano, Psicología Clínica, Psicología Industrial, Docente de la Universidad Técnica de Ambato, Ambato, Ecuador.

${ }^{\text {IV }}$ Magíster en Administración de Empresas Mención Planeación, Ingeniero de Empresas, Abogado de los Tribunales y Juzgados de la República del Ecuador, Docente de la Universidad Técnica de Ambato, Ambato, Ecuador.
} 


\title{
Resumen
}

En la investigación para nuestro artículo científico se determinó como objetivo principal el diseño de nuevas estrategias publicitarias para la decisión de compra del consumidor como repercutió en las empresas comerciales generando un impacto en la sociedad por lo tanto fue necesario crear nuevas estrategias publicitarias que beneficiaron a las empresas con el incremento de sus ventas y al mismo tiempo en captar o atraer nuevos consumidores, para esto se planteó una serie de preguntas alternativas que nos ayudaron a determinar el tipo de estrategias que tuvimos que plantear.

La metodología que se utilizo fue de un paradigma critico propositivo la cual nos permitió realizar una investigación de campo mediante encuestas y poder llegar hasta el lugar de los hechos recaudando importante información por parte del investigador, los resultados esperados fueron óptimos para el desarrollo del estudio y las técnicas a usar en las entrevistas dirigidas a empresas comerciales y consumidores en la provincia de Tungurahua para evaluar el comportamiento que tiene en el mercado. Como resultados tuvimos presentaciones de nuevas estrategias publicitarias que ayudaron a las empresas del sector comercial a incrementar sus ventas y por ende a tener un mejor posicionamiento en la mente del consumidor de tal manera que la empresa se siente satisfecha con las nuevas acciones que se va a seguir. Después de haber estudiado y analizado a diferentes autores y haber cumplido con el objetivo principal se pudo concluir que el diseño de estrategias publicitario es esencial para crear un impacto en la mente del consumidor y por ende a la decisión de compra de nuestros productos.

Palabras clave: estrategias publicitarias; paradigma crítico; decisión de compra.

\begin{abstract}
In the research for our scientific article was determined as the main objective of designing new advertising strategies for the consumer purchase decision as it had an impact on the commercial companies generating an impact on society, therefore it was necessary to create new advertising strategies that benefited the companies with the increase of their sales and at the same time to attract or attract new consumers, for this a series of alternative questions was raised that helped us to determine the type of strategies that we had to raise.
\end{abstract}


The methodology that was used was a critical proactive paradigm which allowed us to carry out a field investigation through surveys and reach the scene of the events, collecting important information from the researcher, the expected results were optimal for the development of the study and the techniques to be used in the interviews directed to commercial companies and consumers in the province of Tungurahua to evaluate the behavior that it has in the market. As a result, we had presentations of new advertising strategies that helped companies in the commercial sector to increase their sales and therefore to have a better position in the mind of the consumer in such a way that the company feels satisfied with the new actions that are going to follow. After having studied and analyzed different authors and having fulfilled the main objective, it was concluded that the design of advertising strategies is essential to create an impact on the mind of the consumer and therefore the decision to purchase our products.

Key words: advertising strategies; critical paradigm; purchase decision.

\section{Resumo}

Na pesquisa para o nosso artigo científico foi determinada como principal objetivo a concepção de novas estratégias de publicidade para a decisão de compra do consumidor e reverberou em empresas comerciais que fazem um impacto na sociedade era, portanto, necessário criar novas estratégias de publicidade que beneficiaram do empresas com o aumento de suas vendas e, ao mesmo tempo, para atrair ou atrair novos consumidores, para isso, foi levantada uma série de questões alternativas que nos ajudaram a determinar o tipo de estratégias que tínhamos que levantar.

A metodologia utilizada foi um paradigma crítico proativo que nos permitiu realizar pesquisa de campo por meio de pesquisas e para chegar ao local coletando informações importantes pelo pesquisador, os resultados esperados foram ideais para o desenvolvimento do estudo e as técnicas a serem utilizadas nas entrevistas dirigidas a empresas comerciais e consumidores na província de Tungurahua para avaliar o comportamento que tem no mercado. Como resultado, tivemos apresentações de novas estratégias de publicidade que ajudaram empresas do setor comercial para aumentar as vendas e, portanto, têm uma melhor posição na mente do consumidor para que a empresa está satisfeita com as novas ações a serem seguir Estamos tendo estudado e analisado diferentes autores e ter cumprido o objetivo principal concluiu-se que o desenho de estratégias de 
publicidade é essencial para criar um impacto na mente dos consumidores e, portanto, a decisão de comprar nossos produtos.

Palavras chave: estratégias publicitárias; paradigma crítico; decisão de compra.

\section{Introducción}

El modelo estratégico publicitario en el comportamiento del consumidor de las empresas del sector comercial ayudara a que las empresas incrementen sus ventas y su utilidad entiendo así primeramente la palabra modelo según (García, 2015) define a la forma que uno propone y sigue en la ejecución de una cosa. Así mismo entendamos la palabra estrategia es el objetivo de la actividad que realiza la dirección de la empresa de manera eficiente. La palabra publicidad según O'Guinn, Allen y Semenik definen al esfuerzo pagado, trasmitido por medios masivos de información con objeto de persuadir" [1], de tal manera decimos que la publicidad es una de las herramientas más poderosas de la mercadotecnia de la promoción que utilizan las empresas. (Thompson, 2014) en donde en los años 50 se empezó a notar como el consumidor compra por impulso, por necesidad. Hay muchas variables para estudiar que van más allá del precio: factores culturales, sociales, personales y psicológicos. Todo esto lleva a estudiar el comportamiento y necesidades de los consumidores según (Malisa, 2013). Es por ello que el comportamiento de los consumidores influye en la decisión de compra del producto.

En la actualidad la situación económica mundial está teniendo efectos claros y significativos sobre la actitud de los consumidores hecho que se refleja en todos los mercados. El índice de confianza de los consumidores ha caído considerablemente, pasando a ocupar las principales preocupaciones de las empresas (Fernandes, Garcia, \& Irene, 2015).

El modelo "estratégico publicitario" es aquel plan de acción diseñado con la finalidad de ayudar a fomentar la venta de determinados productos a los futuros compradores según (Meritxell, 2016) por lo que menciona que es el desarrollo creativo de la publicidad en las estrategias de comunicación. 
La implementación de modelos estratégicos de publicidad en las empresas comerciales ayuda a incrementar las ventas dentro del mercado según (Merino, 2010) permiten también crear una ventaja competitiva.

El modelo estratégico publicitario se la realiza para combatir las amenazas que presentan las empresas. Según (Mendoza, 2017) es por ello que hoy en día no se puede negar la gran influencia de la publicidad, incluso se podría decir y afirmar que la juventud está inmersa en un mundo con un sinfín de productos, en gran parte, el efecto publicitario al que se ha expuesto permite deducir un contexto a futuro, en el que las nuevas generaciones podrían verse expuestos.

La publicidad en las empresas comerciales es de mucha importancia en donde los directivos requieren de nuevas estrategias que logren las metas de ventas con las funciones y apariencia de su sitio web, enfocándose en su target de mercado a través de una elección apropiada del tipo de publicidad, medios y diseño. (Mendoza, 2017)

El problema que se ha detectado en las empresas comerciales según (Calvopiña, 2017) es el bajo nivel del cumplimiento de ventas esto afecta el crecimiento de la empresa, lo que ha generado que las empresa luchen por mantenerse en el mercado; es así que se ha podido analizar que una de sus casusas para que se genere este problema son las limitadas estrategias de publicidad, porque no se las está aplicando de forma correcta lo que da lugar al decremento de las ventas, el mismo que es negativo y es reflejado en los balances, otra de sus causas es que la alta dirección no está capacitada en marketing, porque si lo estuviera podría guiar y su vez evaluar de mejor forma el cumplimiento de objetivos empresariales con la ayuda práctica de la aplicación de estrategias de publicidad, lo cual tiene como efecto las pérdidas financieras. La adquisición innecesaria de mercadería es otra de las causas, que ha conllevado a tener como efecto el sobre stock. Finalmente, se ha detectado que otra de sus causas para que se genere el problema objeto de estudio, es la débil distribución que ocasiona la pérdida de participación en el mercado. En consecuencia, del análisis crítico realizado, se desglosa la necesidad de contar con estrategias de publicidad adecuadas para incrementar las ventas de las empresas del sector comercial.

¿Qué estrategias de publicidad son las más eficientes para incrementar las ventas de las empresas del sector comercial? 
¿De qué manera las estrategias de publicidad ayudaran a incrementar las ventas?

\section{Objetivo de la investigación.}

Determinar un modelo estratégico publicitario en el comportamiento del consumidor de las empresas del sector comercial.

Tienen como finalidad comprender las determinantes del modelo estratégico publicitario en el comportamiento del consumidor cómo influye en la mente del consumir el diseño de estrategias y como nos ayuda a incrementar las ventas. Es por ello que para determinar esta hipótesis es necesario tomar en cuenta, otras investigaciones que proporcionaran información de relevancia.

Se tomó en cuenta a la autora que realizo este artículo científico con el tema: Estrategias de publicidad y su impacto en las ventas de la empresa Repremarva de la ciudad de Ambato.

El principal objetivo a identificar dentro del artículo científico "es diagnosticar las estrategias de publicidad para incrementar las ventas ya que no existe una debida estrategia de publicidad, para promocionar información acerca de las distintas líneas de electrodomésticos existentes dentro del almacén y de esta manera poder dar mejores servicios a los clientes como a los consumidores externos". (Camino, 2015)

La variable de estudio de esta investigación se encuentra relacionada con la variable de nuestro tema que es estrategias publicitarias que van a ayudar a incrementar las ventas de la empresa y por ende mejorar su utilidad.

Otro tema que va ayudar al desarrollo de nuestra investigación es Análisis de Factores publicitarios determinantes en el comportamiento de compra del consumidor del sector comercial de la provincia de Tungurahua en el año 2017.

Como fundamentos importantes podemos señalar que el comportamiento del consumidor es evidente que este evoluciona con el pasar de los años y cada vez presenta nuevas necesidades, es por ello que las empresas obligatoriamente deben estudiar a cada individuo o grupo de individuos a quienes dirigen sus productos y servicios para conocer sus gustos, preferencias y necesidades, para de esta manera diseñar verdaderos satisfactores. (Calvopiña, 2017) 
La relación de la variable Modelo Estratégico Publicitario en el Comportamiento del Consumidor de las empresas del Sector Comercial el estudio de estas variables es indispensable para todas las empresas de acuerdo al estudio con el tema: Análisis del proceso de decisión del consumidor para la estrategia comercial de la empresa.

Hace referencia al consumidor al momento de realizar actividades de compra la conducta del consumidor es importante al momento de comprar ya que él es el que tiene la última palaba al momento de comprar. Ya que el objetivo del marketing es satisfacer a los individuos dentro del sistema económico. (Vivar, 2015)

Como referencia del estudio de las variables encontramos el siguiente tema: Estrategias Publicitarias en el comportamiento del consumidor la cual establece la importancia que tiene el estudio de las estrategias publicitarias en la mente del consumidor las técnicas y la creatividad por parte de los directivos al momento de desarrollar dichas estrategias ayuden a incrementar sus ventas y por lo consiguiente a tener beneficio alguno. (Vega, 2011)

La relación, que guarda la variable Modelo de Estrategias Publicitarias influye mucho en el comportamiento del consumidor la conducta al momento de compra los gustos y preferencias por el producto.

El modelo de "estrategias publicitarias también conocidas como estrategias comerciales, consisten en acciones que se llevan a cabo para lograr un determinado objetivo relacionado con el marketing" en donde las "las estrategias de publicidad, también conocidas como estrategias de mercadotecnia o estrategias de mercadeo consisten en acciones que se llevan a cabo para lograr un determinado objetivo relacionado con el marketing (Ruiz, 2012)”.

El modelo de estratégico publicitario incide en las empresas permitiendo así potenciar el crecimiento de la demanda conquistar nuevos consumidores. Una estrategia debe ser clara, simple, competitiva, positiva original sobre la realización. (Duran, 2014) de acuerdo a estudios realizados sobre modelo estratégico publicitario para las empresas del sector comercial en su ártico científico indica los principales elementos a considerar la situación actual de la empresa, determinación de los objetivos y metas para el diseño el tipo de estrategia de publicidad a utilizar. 
Dentro de las propiedades y características de la variable podemos decir según (Fernández \& Gracia, 2016) estrategias para la promoción de ventas impulsan al cliente a la compra, estrategias de posicionamiento de la mente del consumidor, estrategias competitivas.

De esta manera impulsan a los consumidores a tomar la decisión de compra de un determinado producto.

Según (Ruiz, 2012) el comportamiento del consumidor implica un proceso es decir una sucesión de etapas que permiten conocer las "causas, variaciones y resultados de las acciones de consumo estos procesos que caracterizan al comportamiento del consumidor".

El comportamiento del consumir influye mucho dentro de las empresas ya que es el proceso de dación para adquirir el producto y satisfacer nuestras necesidades.

Dentro del comportamiento del consumidor en empresas comerciales, un estudio reciente de la Universidad Nacional del Cuyo realizada por (Malisa, 2013) sobre "el comportamiento del consumidor actual en las empresas comerciales" hace referencia a los resultados más resaltantes de esta investigación el comportamiento del consumidor en la toma de decisiones "Las actitudes y las percepciones según el posicionamiento que tengan en el consumidor "El consumidor forma actitudes hacia los productos y estos influyen favorable o desfavorablemente en la decisión de compra.

Nos queda claro que el comportamiento del consumidor influye en la decisión de compra del producto es por ello que esta investigación se encarga de estudiar los factores que intervienen al momento de comprar.

Las propiedades y características del comportamiento del consumidor la publicidad es una técnica comercial que puede definirse como una comunicación masiva pagada, cuyo propósito final es el divulgar la información, producir o cambiar actitudes. De esta manera la publicidad trata de llamar la atención del público sobre el producto anunciado e intenta impactar mediante la estética de los anuncios. 


\section{Materiales y Métodos}

Para este artículo vamos a utilizar un paradigma crítico propositivo ya que nos permite utilizar la crítica reflexiva en los diferentes procesos teniendo un enfoque inductivo - deductivo y cualitativo - cuantitativo, ya que la investigación será de campo tomando un muestreo que involucra directamente a las diferentes empresas comerciales existentes en la provincia de Tungurahua.

Una vez definida la población objeto de estudio tomamos una parte de la misma, que se denomina muestra, por lo general se hace uso de esta herramienta cuando el número de integrantes de la población es muy grande. El enfoque cuantitativo busca que los resultados obtenidos de la muestra se trasladen o representen al total de la población; mientras tanto el enfoque cualitativo no necesita que los resultados representan en la población y consideran sub grupos dentro de la misma, es en este momento analiza por qué la presente investigación es carácter cualitativo - cuantitativo a que considera dos grupos de análisis como son los interesados y no interesados.

El instrumento que se aplicara para la investigación de campo será un cuestionario el cual constara de 9 preguntas, ya que nos permitirá a obtener información según nuestro objetivo; el cuestionario será descriptivo creando una serie de repuestas estándar en el cual solo se escogerá una sola opción.

Para la elaboración de las preguntas tomamos en cuenta nuestras variables y las opiniones de algunos autores la cual se verá reflejada en la siguiente tabla.

\begin{tabular}{|c|c|}
\hline VARIABLES & DIMENSIONES \\
\hline \multicolumn{2}{|c|}{ MODELO ESTRATÉGICO PUBLICITARIO } \\
\hline $\begin{array}{l}\text { Podemos decir que el modelo de } \\
\text { "estrategias publicitarias también conocidas } \\
\text { como estrategias comerciales, consisten en } \\
\text { acciones que se llevan a cabo para lograr un } \\
\text { determinado objetivo relacionado con el } \\
\text { marketing" en donde las "las estrategias de }\end{array}$ & $\begin{array}{l}\text { - El modelo de estrategias publicitarias } \\
\text { incide en las empresas permitiendo así } \\
\text { potenciar el crecimiento de la demanda } \\
\text { y conquistar nuevos consumidores. } \\
\text { - La publicidad como elemento con } \\
\text { utilidad. } \\
\text { - La publicidad dando la vuelta al }\end{array}$ \\
\hline
\end{tabular}


publicidad, también conocidas como estrategias de mercadotecnia o estrategias de mercadeo consisten en acciones que se llevan a cabo para lograr un determinado objetivo relacionado con el marketing (Ruiz, 2012)". mundo con mensajes diferente.

- El modelo estratégico publicitario se la realiza para combatir las amenazas que presentan las empresas.

\section{COMPORTAMIENTO DEL CONSUMIDOR}

Según (Ruiz, 2012) el comportamiento del consumidor implica un proceso es decir una sucesión de etapas que permiten conocer las "causas, variaciones $y$ resultados de las acciones de consumo estos procesos que caracterizan al comportamiento del consumidor".
- Las actitudes y las percepciones según el posicionamiento que tenga en el consumidor.

- El consumidor forma actitudes hacia los productos y esto influye favorable o desfavorablemente en la decisión de compra.

FUENTE: (Ruiz, 2012), (Fernández \& Gracia, 2016), (Malisa, 2013).

\section{ELABORADO POR: Zambrano Salazar Carmen}

Una vez analizados y comparados los criterios de los autores se realizó la elaboración de las preguntas las cuales tuvieron un lenguaje sencillo, claro, alcanzable y relevante con el fin de cumplir con el objetivo establecido.

\section{Resultados}

Se realizaron 384 encuestas en las diferentes empresas de la provincia de Tungurahua con un total de 9 preguntas las cuales tuvieron los siguientes resultados.

Tabla 1: Diseño de estrategias para publicidad.

\begin{tabular}{|c|c|c|c|c|c|}
\hline & & Frecuencia & Porcentaje & $\begin{array}{c}\text { Porcentaje } \\
\text { válido }\end{array}$ & $\begin{array}{l}\text { Porcentaje } \\
\text { acumulado }\end{array}$ \\
\hline \multirow[t]{5}{*}{ Válido } & Siempre & 238 & 62,0 & 62,0 & 62,0 \\
\hline & Casi Siempre & 107 & 27,9 & 27,9 & 89,8 \\
\hline & Casi nunca & 36 & 9,4 & 9,4 & 99,2 \\
\hline & Casi Nunca & 3 &, 8 &, 8 & 100,0 \\
\hline & Total & 384 & 100,0 & 100,0 & \\
\hline
\end{tabular}

Elaborado por: Zambrano Carmen.

Fuente: Encuesta (2018). 


\section{Interpretación:}

Se pudo determinar que: $62 \%$ encuestados corresponde a siempre, $27.9 \%$ a casi siempre, $9.4 \%$ casi nunca y $0.8 \%$ nunca, por lo tanto, la mayoría de los encuestados si consideran necesario aplicar un diseño de estrategias de publicidad en el sector comercial.

Tabla 2: Estrategias Competitivas.

\begin{tabular}{|ll|l|l|l|l|}
\hline & & Frecuencia & Porcentaje & $\begin{array}{c}\text { Porcentaje } \\
\text { válido }\end{array}$ & $\begin{array}{l}\text { Porcentaje } \\
\text { acumulado }\end{array}$ \\
\hline Válido & Siempre & 179 & 46,6 & 46,6 & 46,6 \\
& Casi siempre & 152 & 39,6 & 39,6 & 86,2 \\
En Ocasiones & 42 & 10,9 & 10,9 & 97,1 \\
Casi Nunca & 9 & 2,3 & 2,3 & 99,5 \\
Nunca & 2 &, 5 &, 5 & 100,0 \\
Total & 384 & 100,0 & 100,0 & \\
\hline
\end{tabular}

Elaborado por: Zambrano Carmen.

Fuente: Encuesta (2018).

\section{Interpretación:}

El $46.6 \%$ siempre, $39.6 \%$ casi siempre, $10.9 \%$ en ocasiones, $2.3 \%$ casi nunca y $0.5 \%$ nunca, se dedujo que las estrategias competitivas sin son necesarias para el sector comercial para poder incrementar las ventas.

Tabla 3: Estrategias Publicitarias

\begin{tabular}{|cc|c|c|c|c|}
\hline & Frecuencia & Porcentaje & $\begin{array}{c}\text { Porcentaje } \\
\text { válido }\end{array}$ & $\begin{array}{c}\text { Porcentaje } \\
\text { acumulado }\end{array}$ \\
\hline Válido & Siempre & 135 & 35,2 & 35,2 & 35,2 \\
& Casi siempre & 153 & 39,8 & 39,8 & 75,0 \\
En Ocasiones & 83 & 21,6 & 21,6 & 96,6 \\
Casi Nunca & 12 & 3,1 & 3,1 & 99,7 \\
Nunca & 1 &, 3 &, 3 & 100,0 \\
Total & 384 & 100,0 & 100,0 & \\
\hline
\end{tabular}

Elaborado por: Zambrano Carmen.

Fuente: Encueta (2018). 


\section{Interpretación:}

El $35.2 \%$ siempre, $39.8 \%$ casi siempre, $21.6 \%$ en ocasiones, $3.1 \%$ casi nunca y $0.3 \%$ nunca supieron manifestar que la mayoría si aplican estrategias publicitarias para incrementar las ventas de sus productos.

Tabla 4: Promoción por la Empresa

\begin{tabular}{|c|c|c|c|c|c|}
\hline & & Frecuencia & Porcentaje & $\begin{array}{l}\text { Porcentaje } \\
\text { válido } \\
\end{array}$ & $\begin{array}{l}\text { Porcentaje } \\
\text { acumulado }\end{array}$ \\
\hline \multirow[t]{6}{*}{ Válido } & Siempre & 115 & 29,9 & 29,9 & 29,9 \\
\hline & Casi Siempre & 111 & 28,9 & 28,9 & 58,9 \\
\hline & En Ocasiones & 117 & 30,5 & 30,5 & 89,3 \\
\hline & Casi Nunca & 30 & 7,8 & 7,8 & 97,1 \\
\hline & Nunca & 11 & 2,9 & 2,9 & 100,0 \\
\hline & Total & 384 & 100,0 & 100,0 & \\
\hline
\end{tabular}

Elaborado por: Zambrano Carmen.

Fuente: Encuesta (2018).

\section{Interpretación:}

Se pudo conocer quienes ha recibido promoción por parte de las empresas; $29.9 \%$ siempre, $28.9 \%$ casi siempre, $30.5 \%$ en ocasiones, $7.8 \%$ casi nunca y $2.9 \%$ nunca por lo tanto, la mayor parte de las empresas ofrece algún tipo de promoción a sus clientes.

Tabla 5. Medios de Comunicación

\begin{tabular}{|c|c|c|c|c|c|}
\hline & & Frecuencia & Porcentaje & $\begin{array}{c}\text { Porcentaje } \\
\text { válido }\end{array}$ & $\begin{array}{l}\text { Porcentaje } \\
\text { acumulado }\end{array}$ \\
\hline \multirow[t]{6}{*}{ Válido } & Siempre & 165 & 43,0 & 43,0 & 43,0 \\
\hline & Casi Siempre & 135 & 35,2 & 35,2 & 78,1 \\
\hline & En Ocasiones & 73 & 19,0 & 19,0 & 97,1 \\
\hline & Casi Nunca & 7 & 1,8 & 1,8 & 99,0 \\
\hline & Nunca & 4 & 1,0 & 1,0 & 100,0 \\
\hline & Total & 384 & 100,0 & 100,0 & \\
\hline
\end{tabular}

Elaborado por: Zambrano Carmen.

Fuente: Encuesta (2018). 


\section{Interpretación:}

Se pudo conocer que, $43 \%$ siempre, $35.2 \%$ casi siempre, $19 \%$ en ocasiones, $1.8 \%$ casi nunca y $1 \%$ nunca, por lo tanto la mayoría consideran que si se debería realizar mayor publicidad en los medios de comunicación para darse conocer en el mercado con sus respectivos productos.

Tabla 6: Personal Calificado.

\begin{tabular}{|c|c|c|c|c|c|}
\hline & & Frecuencia & Porcentaje & $\begin{array}{c}\text { Porcentaje } \\
\text { válido }\end{array}$ & $\begin{array}{l}\text { Porcentaje } \\
\text { acumulado }\end{array}$ \\
\hline \multirow[t]{6}{*}{ Válido } & Siempre & 103 & 26,8 & 26,8 & 26,8 \\
\hline & Casi Siempre & 126 & 32,8 & 32,8 & 59,6 \\
\hline & En Ocasiones & 108 & 28,1 & 28,1 & 87,8 \\
\hline & Casi Nunca & 37 & 9,6 & 9,6 & 97,4 \\
\hline & Nunca & 10 & 2,6 & 2,6 & 100,0 \\
\hline & Total & 384 & 100,0 & 100,0 & \\
\hline
\end{tabular}

Elaborado por: Zambrano Salazar.

Fuente: Encuesta (2018).

\section{Interpretación:}

Se pudo conocer la cantidad de personas calificadas al momento del desarrollo de estrategias; $26.8 \%$ siempre, $32.8 \%$ casi siempre, $28.1 \%$ en ocasiones, $9.6 \%$ casi nunca y $2.6 \%$ nunca por consiguiente la mayoría de las empresas cuentan con personal calificado para dicha elaboración.

Tabla 7: Frecuencia de Compra.

\begin{tabular}{|c|c|c|c|c|c|}
\hline & & Frecuencia & Porcentaje & $\begin{array}{c}\text { Porcentaje } \\
\text { válido }\end{array}$ & $\begin{array}{l}\text { Porcentaje } \\
\text { acumulado }\end{array}$ \\
\hline \multirow[t]{5}{*}{ Válido } & Siempre & 155 & 40,4 & 40,4 & 40,4 \\
\hline & Casi Siempre & 147 & 38,3 & 38,3 & 78,6 \\
\hline & En Ocasiones & 76 & 19,8 & 19,8 & 98,4 \\
\hline & Casi Nunca & 6 & 1,6 & 1,6 & 100,0 \\
\hline & Total & 384 & 100,0 & 100,0 & \\
\hline
\end{tabular}

Elaborado por: Zambrano Carmen.

Fuente: Encuesta (2018) 


\section{Interpretación:}

Pudimos concluir que; $40.4 \%$ siempre, $38.3 \%$ casi siempre, $19.8 \%$ en ocasiones, $1.6 \%$ casi nunca; la mayoría compran con frecuencia determinado producto.

Tabla 8: Factores de Compra

\begin{tabular}{|c|c|c|c|c|c|}
\hline & & Frecuencia & Porcentaje & $\begin{array}{l}\text { Porcentaje } \\
\text { válido }\end{array}$ & $\begin{array}{l}\text { Porcentaje } \\
\text { acumulado }\end{array}$ \\
\hline \multirow[t]{6}{*}{ Válido } & Marca & 87 & 22,7 & 22,7 & 22,7 \\
\hline & Calidad & 165 & 43,0 & 43,0 & 65,6 \\
\hline & Variedad & 44 & 11,5 & 11,5 & 77,1 \\
\hline & Precio & 85 & 22,1 & 22,1 & 99,2 \\
\hline & Otro & 3 &, 8 &, 8 & 100,0 \\
\hline & Total & 384 & 100,0 & 100,0 & \\
\hline
\end{tabular}

Elaborado por: Zambrano Carmen.

Fuente: Encuesta (2018).

\section{Interpretación:}

E1 $22.7 \%$ marcas, $43 \%$ calidad, $11.5 \%$ variedad, $22.1 \%$ precio, $0.8 \%$ otros; la mayoría hicieron énfasis en la calidad al momento de realizar una compra de un determinado producto.

Tabla 9: Proceso de Decisión.

\begin{tabular}{|c|c|c|c|c|c|}
\hline & & Frecuencia & Porcentaje & $\begin{array}{c}\text { Porcentaje } \\
\text { válido }\end{array}$ & $\begin{array}{l}\text { Porcentaje } \\
\text { acumulado }\end{array}$ \\
\hline \multirow[t]{7}{*}{ Válido } & Atención & 113 & 29,4 & 29,4 & 29,4 \\
\hline & Calidad & 144 & 37,5 & 37,5 & 66,9 \\
\hline & Servicio & 40 & 10,4 & 10,4 & 77,3 \\
\hline & Promociones & 45 & 11,7 & 11,7 & 89,1 \\
\hline & Descuento & 40 & 10,4 & 10,4 & 99,5 \\
\hline & Otros & 2 & ,5 & ,5 & 100,0 \\
\hline & Total & 384 & 100,0 & 100,0 & \\
\hline
\end{tabular}

Elaborado por: Zambrano Carmen.

Fuente: Encueta (2018)

\section{Interpretación:}

El valor más relevante al momento de realizar una compra nos dio como resultado; $29.4 \%$ atención, $37.5 \%$ calidad, $10.4 \%$ servicios, $11.7 \%$ promociones, $10.4 \%$ descuentos y $0.5 \%$ otros; 
dando a conocer que en la mayoría de los encuestados predomina el valor de la calidad en los productos.

\section{Discusión}

Mediante análisis e interpretaciones se llegó a comprender la importancia que tiene determinar un modelo estratégico publicitario que pueda influir en el comportamiento del consumidor de las empresas comerciales de la provincia de Tungurahua.

Solo el $62 \%$ de los encuestados en las diferentes empresas comerciales de la provincia de Tungurahua están consiente de la necesidad de un diseño estratégico de publicidad y la importancia que tiene esta ya que nos permitirá incrementar las ventas y su utilidad debido a que la situación económica mundial nos ha convertido en un mundo competitivo por lo cual debemos estar a la vanguardia con nuestros productos; mientras que el 27,9\% no están muy convencido de la aplicación de dicho diseño creando la inquietud de por qué será y a la vez dando paso a las siguientes preguntas ¿En que nos beneficia? ¿No se cómo aplicarlo? Pero el 10.2\% por la falta de capacitación, información o preparación desconocen en su totalidad la necesidad de la aplicación de un diseño de estrategias de publicidad.

Si hablamos de la importancia y la necesidad de crear un diseño estratégico de publicidad el cual permita influir en el comportamiento del consumidor solo el 35.2\% y 39.8\% de las empresas comerciales de la provincia de Tungurahua, siempre y casi siempre aplica, dichas estrategias, ya que ellos comprenden que es una herramienta para alcanzar sus objetivos y así lograr un mensaje concreto que se quiere hacer llegar al consumidor y verse beneficiado con el incremento de sus ventas y posteriormente con las utilidades que genera.

Para tener resultados favorable al momento de diseñar y aplicar un modelo estratégico publicitario que pueda influir en el comportamiento del consumidor es necesario contar con personas calificadas para dicho desarrollo de estrategias ya que es un proceso donde involucran muchas variables pero solo el $26.8 \%$ y $32.8 \%$ siempre y casi siempre cuentan con personas calificadas para la realización y aplicación de las estrategias, en definitiva manejar con claridad los objetivos establecidos es fundamental para el cumplimiento de los mismos. 
A continuación presentamos una tabla de correlación de las viables la cual nos indica la relación de una con la otra:

\begin{tabular}{|c|c|c|c|c|c|c|c|}
\hline & $\begin{array}{l}\text { Diseño de } \\
\text { estrategias } \\
\text { publicitarios. }\end{array}$ & $\begin{array}{l}\text { Estrategias } \\
\text { competitivas }\end{array}$ & $\begin{array}{l}\text { Aplicación } \\
\text { estrategias } \\
\text { competitivas }\end{array}$ & $\begin{array}{l}\text { Promoción } \\
\text { por la } \\
\text { empresa. }\end{array}$ & $\begin{array}{l}\text { Mayor } \\
\text { publicidad en } \\
\text { medios de } \\
\text { comunicación }\end{array}$ & $\begin{array}{l}\text { Personal } \\
\text { calificado }\end{array}$ & $\begin{array}{l}\text { Frecuencia } \\
\text { de copras }\end{array}$ \\
\hline $\begin{array}{l}\text { Diseño de estrategias } \\
\text { publicitarias. }\end{array}$ & 1,000 &, $444^{* *}$ &, $278^{* *}$ &, $226^{* *}$ &, $319^{* *}$ &, $207^{* *}$ &, $119^{*}$ \\
\hline $\begin{array}{l}\text { Estrategias } \\
\text { competitivas }\end{array}$ &, $444^{* *}$ & 1,000 &, $208^{* *}$ & 0,084 &, $252^{* *}$ &, $164^{* *}$ & 0,090 \\
\hline $\begin{array}{l}\text { Aplicación } \\
\text { estrategias } \\
\text { competitivas }\end{array}$ &, $278^{* *}$ &, $208^{* *}$ & 1,000 &, $376^{* * *}$ &, $151^{* *}$ &, $324^{* * *}$ &, $196^{* *}$ \\
\hline $\begin{array}{l}\text { Promoción por la } \\
\text { empresa }\end{array}$ &, $226^{* *}$ & 0,084 &, $376^{* *}$ & 1,000 &, $148^{* *}$ &, $371^{* *}$ &, $123^{*}$ \\
\hline $\begin{array}{l}\text { Mayor publicidad en } \\
\text { Medios de } \\
\text { comunicación }\end{array}$ &, $319^{* *}$ &, $252^{* *}$ &, $151^{* *}$ &, $148^{* *}$ & 1,000 & 0,076 &, $176^{* *}$ \\
\hline Personal calificado &, $207^{* *}$ &, $164^{* *}$ &, $324^{* *}$ &, $371^{* *}$ & 0,076 & 1,000 &, $133^{* *}$ \\
\hline $\begin{array}{l}\text { Frecuencia de } \\
\text { compra. }\end{array}$ &, $119^{*}$ & 0,090 &, $196^{* *}$ &, $123^{*}$ &, $176^{* *}$ &, $133^{* *}$ & 1,000 \\
\hline
\end{tabular}

Elaborado por: Zambrano Carmen.

Fuente: Encuesta (2018)

Observamos que hay correlación entre la necesidad de crear un diseño de estrategias publicitarias y la variable de aplicar estrategias publicitarias competitivas en las empresas comerciales las cuales nos permiten proponer una serie de estrategias y tácticas que lleve a cabo el cumplimiento del objetivo propuesto, para esto debemos de tener en cuenta las ventaja y desventajas del producto y del mercado a quien va dirigido con el fin de incrementar las ventas, crear un posicionamiento en la mente del consumidor, captar nuevos consumidores y estar a la vanguardia.

Al momento de diseñar, aplicar o desarrollar el modelo estratégico de publicidad en el comportamiento del consumidor del sector comercial en la provincia de Tungurahua, deberá ser realizado por un personal calificado ya que deberá de contar con las destrezas o habilidades que le permita llevar a cabo con eficiencia las estrategias diseñada y cumplir con el objetivo propuesto. 


\section{Conclusiones}

De conformidad con los resultados de las encueta y la correlación de las variables aplicadas a las diferentes empresas del sector comercial de la provincia de Tungurahua y objetivos del Artículo Científico, se concluye.

En la actualidad las estrategias publicitarias hace parte de las decisiones de compras del consumidor, ya que repercuten generando un posicionamiento en la mente del consumidor trasladando el mensaje de una forma clara y concisa con la finalidad de hacer sentir diferentes emociones y quieran experimentar la decisión de compra.

La mayoría de los encuestados claramente entienden la importancia de la aplicación de una estrategia publicitaria pero en donde debemos hacer énfasis es en cómo pueden aplicarla ya que existen diferentes estrategias según lo que se quiera conseguir, si es por el lado del producto debemos tomar encueta si es nuevo, si es una imitación, si está en declive o si se quiere captar o atraer a nuevos consumidores o pretender que nuestros clientes actuales consuman con más frecuencias nuestros productos, dependiendo de los objetivos se aplicara o diseñara la estrategia publicitaria correspondiente.

Nombraremos algunas de las estrategias publicitarias que ayudaran incrementar las ventas y a la vez que influya en la decisión de compra.

Se implementó una estrategia publicitaria competitiva, ayudara a mostrar las ventajas de los productos ante la competencia el cual creara un posicionamiento en la mente del consumidor ira acompañado de promociones agresivas que ayudara incrementar el consumo del producto.

Se realizó una estrategia de desarrollo Extensiva: para captar nuevos consumidores fortaleciendo la maraca, servicios que se brinda, canales de distribución y redes sociales Intensivas: ayudara a mantener y fidelizar a los consumidores actuales y aumentar su consumo, ofreciendo cupones de descuentos, regalos por monto de compra.

\section{Referencias Bibliográficas}


Fernandes, E., Garcia, D., \& Irene, M. (20 de 09 de 2015). Las estrategias publicitarias a nivel mundial. Obtenido de https://core.ac.uk/download/pdf/61396499.pdf

Calvopiña, G. (2017). Factores publicitarios determinantes en el comportamiento de compra del consumidor del sector comercial. Obtenido de http://repositorio.uta.edu.ec/bitstream/123456789/26279/1/570\%20MKT.pdf

Camino, J. (15 de 03 de 2015). "Estrategias de publicidad y su impacto en las ventas de la empresa Repremarva de la ciudad de Ambato durante el año2012. Obtenido de http://repositorio.uta.edu.ec/bitstream/123456789/7585/1/141\%20MKT.pdf

\section{Doris Martínez. (2016). FACTORES CLAVE EN MARKETING ENFOQUE EMPRESAS DE} SERVICIOS. Obtenido de http://www.redalyc.org/pdf/709/70946593003.pdf

Duran, A. (2014). Publicado. Obtenido de http://www.albertodeduran.es/wpcontent/uploads/2014/08/2x02-Planificaci\%C3\%B3n-estrat\%C3\%A9gica-publicitaria.pdf

Fernández, E., \& Gracia, D. (2016). Las estrategias publicitarias. Obtenido de https://core.ac.uk/download/pdf/61396499.pdf

Garcia, L. (2015). Concepto de Modelo. Obtenido de http://www.eumed.net/tesisdoctorales/2012/1sg/concepto_modelo.html

Jenny Camino. (2014). Estrategias de publicidad y su impacto en las ventas de la Empresa Repremarva de la ciudad de Ambato, durante el año 2014. Obtenido de http://repositorio.uta.edu.ec/bitstream/123456789/7585/1/141\%20MKT.pdf

Malisa, D. (2013). El comportamiento del consumidor actual. Obtenido de http://bdigital.uncu.edu.ar/objetos_digitales/8046/raiteri-melisa-daniela.pdf

María Tobar. (2013). La toma de decisiones en el mundo del consumo basado en procesos cerebrales. Obtenido de http://repositorio.usfq.edu.ec/bitstream/23000/2158/1/106124.pdf 
Mendoza, L. (2017). Publicidad en las empresas. Obtenido de http://www.revistasbolivianas.org.bo/scielo.php?pid=S252127372017000100006\&script=sci_arttext

Merino, J. (28 de 11 de 2010). La investigación de mercados. Obtenido de https://eprints.ucm.es/11230/1/La_Investigaci\%C3\%B3n_de_Mercados_en_la_Empresa.pdf

Meritxell, J. (20 de 10 de 2016). La planificación estratégica en las agencias de publicidad. Obtenido de http://www.colpublirp.com/wp-content/uploads/2014/04/2n-Premi_CAST-OK.pdf

Pablo Arcos. (2014). ANÁLISIS DEL COMPORTAMIENTO DEL CONSUMIDOR EN LA COMPRA DE TABLETS EN LA CIUDAD DE QUITO, PARA PERSONAS ENTRE LOS 18 A 40 AÑOS DE EDAD, EN EL AÑO 2013. Obtenido de http://repositorio.puce.edu.ec/bitstream/handle/22000/7816/2.22.000818.pdf;sequence=4

Ruiz, S. (2012). El comportamiento del consumidor en marketing. Obtenido de http://bibliotecadigital.univalle.edu.co/bitstream/10893/2066/1/El\%20comportamiento\%20del\%2 0consumidor $\% 20$ en $\% 20$ marketing2.pdf

Sonia Ottaviano. (2015). ANÁLISIS DEL IMPACTO DE LA PUBLICIDAD EN LAS DECISIONES DE CONSUMO EN LOS NIÑOS.

Thompson, I. (2014). Definición de Publicidad. Obtenido de http://brd.unid.edu.mx/recursos/PUBLICIDAD/BLOQUE1/Lecturas/1.3\%20Puyblicidad.\%20Sus $\% 20 d e f i n i c i o n e s . p d f$

Vega, P. (2011). Estrategias Publicitarias en la Decisión de Compra. Obtenido de http://www.redalyc.org/pdf/729/72923962002.pdf

Vivar, L. (2015). Análisis del proceso de decisión del consumidor. Obtenido de file://C:/Users/SDO/Documents/Dialnet-

AnalisisDelProcesoDeDecisionDelConsumidorParaLaEst-786117.pdf 
Webloyalty y Oxford Brookes University. (2017). Solo marketing. Obtenido de https://solomarketing.es/5-motivos-que-llevan-a-los-consumidores-a-tomar-la-decision-decompra/ 